\title{
SARS-CoV-2 variants of concern: implications on the second wave of COVID-19 in India
}

\author{
Ian Christopher N. Rocha1, Samarth Goyal'2, Sudhan Rackimuthu3 ${ }^{3}$, Shubhika Jain² \\ ${ }^{1}$ School of Medicine, Centro Escolar University, Manila, Philippines; \\ ${ }^{2}$ Kasturba Medical College, Manipal, Karnataka, India; \\ ${ }^{3}$ Father Muller Medical College, Mangalore, Karnataka, India
}

Article received 15 May, 2021; accepted 16 June, 2021

Dear Editor,

Coronavirus disease 2019 (COVID-19), which was

first detected in Wuhan, China in December 2019, became a pandemic in March 2020 due to its rapid global spread, reaching 220 countries and territories with $159,628,512$ confirmed cases and 3,318,439 deaths as of May 10, 2021 [1]. India, being the second most populous country, became the second worst-hit country in the world in terms of total number of COVID-19 cases.

Since its first case was identified in Kerala, various measures including a complete lockdown, augmented and magnified testing, and travel prohibitions were taken up as a response to increasing daily count. Despite all these actions, more than 0.7 million cases were reported by July 2020 in India which later peaked in mid-September with the maximum cases recorded to be 97,859 on September 16, 2020 with alarming repercussions on all the sectors of the country [1]. Following this, a fall in the number of cases and flattening of the curve was observed in the country.

Unfortunately, a rise in the number of cases again has been noticed since April 2021. The daily count peaked at 414,433 on May 6, 2021, which is the

Corresponding author

Ian Christopher N. Rocha

E-mail: rocha1750018@ceu.edu.ph highest reported number of daily new cases in the country so far since the start of the pandemic, indicating the peak of the second wave [1]. Indian states under maximum scrutiny are Maharashtra, Karnataka, Kerala, Uttar Pradesh, Tamil Nadu, Delhi, and Andhra Pradesh with 4,996,758, 1,838,885, 1,824,856, 1,453,679, 1,323,965, 1,292,867, and 1,245,374 cases, respectively [1]. As of May 10, 2021, there have been 22,992,517 confirmed cases of COVID-19 with 250,025 deaths [1]. India accounts for $14.4 \%$ of the total cases globally.

This sudden surge has been a consequence of infringement of the stringent measures which once brought the situation under control, such as social distancing, hand washing, wearing masks, and closing up of social places. In addition to this, events like the Kumbh Mela festival, elections, and rallies have resulted in gatherings of uncountable masses. Another potential cause that has been reported is the mutating virus and the new variants found in the Indian population. Viral agents tend to undergo conformational change leading to mutations as they travel from one person to another. Although these changes are frequent, usually they are insignificant [2]. Significant alterations in characteristics such as more infectivity, resistance to vaccines, ability to escape immune surveillance are brought by mutations that alter the spike protein $[2,3]$.

The mutated variants of severe acute respiratory syndrome coronavirus 2 (SARS-CoV-2) are cate- 
gorized into the following three classifications [3]. Variants of interest (VOI) are variants with diminished neutralization by antibodies produced by past infections or inoculation, reduced treatment effectiveness, potential diagnostic effects, and projected rise in disease transmission or severity. Variants of concern (VOC), on the other hand, have higher evidence of negative impacts on testing, therapeutics, and vaccines, as well as a higher risk of disease transmission and severity than the VOIs. Lastly, variant of high consequence has conclusive evidence that preventive measures and medical treatments are no longer effective in dealing with its effects [3].

In the context of India's second wave of COVID-19 pandemic, the appearance of the B.1.617+ variant, a newly identified double mutant variant that was first detected in the country, undoubtedly exacerbated the uncontrollable and devastating COVID-19 situation in India [4]. As of May 10, 2021, the Indian double mutant variant is already declared as a VOC by the World Health Organization due to its high transmissibility, reduced neutralization, and rapid global spread reaching 44 countries and territories [4, 5]. Additionally, the arrival of three out of six known VOCs, such as B.1.1.7 variant from the United Kingdom (UK), B.1.351 variant from South Africa, and P.1 variant from Brazil, and the entry of one VOI, such as B.1.525 from the UK and Nigeria, have all contributed to the exponential rise of COVID-19 cases in the country [5].

Currently, there is a lot to be learned about the characteristics of the various variants of SARS$\mathrm{CoV}-2$, but researchers are working hard to help provide valuable information that may prove pivotal in tackling the crisis. However, the following suggestions can help in curbing the spread of the transmissible strains. The important acts of prevention such as using facemasks, maintaining physical distance, and hand hygiene continue to be at the forefront of preventing the spread of COVID-19. It is also extremely important to not be affected by pandemic fatigue and to continue to stay vigil and adhere to public health guidelines and advice.

It is imperative to understand that vaccination still plays a crucial role in managing the pandemic as vaccine efficacy has been proven to reduce COVID-19. However, only $9.7 \%$ of the country's population has been inoculated with at least one dose of COVID-19 and just 2.5\% has been fully vaccinated as of May 9, 2021, despite being one of the largest sources of COVID-19 vaccines globally $[6,7]$. Hence, pushing for faster vaccination of all individuals, just like in other countries with successful vaccination programs, will reduce the burden on the healthcare system and possibly also provide some amount of protection against the variants $[8,9]$. Future targeted research can lead to a change in the composition of the vaccines to be more effective against the mutated strains.

Stern implementation and a further bolstering of surveillance strategies are vital to help identify and contain mutant variants of SARS-CoV-2. Whole-genome sequencing (WGS) in patients suspected to have been infected by a variant and those being tested positive with recent travel history from confirmed hot spots can lead to timely detection and effective management. Prospective surveillance with the help of WGS in a small proportion of patients testing positive from different states may also help to identify variants if any are present [10]. International and local collaboration of research groups and sharing of sequenced data of SARS-CoV-2 viruses will help one another counter the incessantly transforming pandemic through continuous monitoring.

\section{Conflict of interest}

The authors declare that there is no conflict of interest.

\section{Funding}

None

\section{REFERENCES}

[1] \#IndiaFightsCorona COVID-19. Available at: https:/ / www.mygov.in/covid-19/ [Accessed 11 May 2021].

[2] Biswas S. 'Double mutant': what are the risks of India's new COVID-19 variant. BBC News. 25 Mar 2021. Available at: https://www.bbc.com/news/world-asiaindia-56517495 [Accessed May 2021].

[3] SARS-CoV-2 variant classifications and definitions. Centers for Disease Control and Prevention. 5 May 2021. Available at: https://www.cdc.gov/coronavirus / 2019-ncov / cases-updates / variant-surveillance / variant-info.html [Accessed 8 May 2021].

[4] B.1.617 variant of concern at global level: WHO. Hindustan Times. 11 May 2021. Available at: https:// www.hindustantimes.com/india-news/b1617-variant- 
of-concern-at-global-level-who-101620675440094.html [Accessed 11 May 2021].

[5] Tracking of variants. GISAID. Available at: https:/ / www.gisaid.org/hcov19-variants/ [Accessed 11 May 2021].

[6] Mathieu E, Ritchie H, Ortiz-Ospina E, et al. A global database of COVID-19 vaccinations. Nat Hum Behav. 2021. doi: 10.1038/s41562-021-01122-8.

[7] Chakraborty C, Sharma AR, Bhattacharya M, Lee SS, Agoramoorthy G. COVID-19 vaccine: challenges in developing countries and India's initiatives. Infez Med. 2021; 29 (1), 165-6.

[8] Rocha ICN. Employing medical anthropology ap- proach as an additional public health strategy in promoting COVID-19 vaccine acceptance in Bhutan. Int J Health Plann Manage. 2021. doi: 10.1002/hpm.3191.

[9] The effects of virus variants on COVID-19 vaccines. World Health Organization. 1 Mar 2021. Available at: https: / / www.who.int/news-room/feature-stories / detail/the-effects-of-virus-variants-on-covid-19-vaccines [Accessed 10 May 2021].

[10] Genomic surveillance for SARS-CoV-2 variants. Centers for Disease Control and Prevention. 2 Apr 2021. Available at: https://www.cdc.gov/coronavirus / 2019-ncov / cases-updates/variant-surveillance. html [Accessed 10 May 2021]. 